\title{
Representações discursivas surdas no contexto do distanciamento social da pandemia da Covid-19
}

\author{
Deaf Discursive Representations in the Context of the Social \\ Distancing of Covid-19 pandemic
}

\author{
Marília do Socorro Oliveira Araújo ${ }^{1}$ \\ Márcia Monteiro Carvalho ${ }^{2}$ \\ Rosângela do Socorro Nogueira de Sousa ${ }^{3}$
}

\begin{abstract}
Resumo: Trata-se de uma discussão a respeito da vivência sob a medida do distanciamento social a partir da percepção de pessoas surdas e de uma pessoa surdocega. Objetivou-se analisar as representações discursivas da pessoa surda e surdocega acerca das medidas de distanciamento social, cujo resultado caracteriza um isolamento, seja ele anterior e/ou durante a pandemia da Covid-19. Teoricamente, o trabalho se baseia no conceito de Representação Social (MOSCOVICI, 2007) e, metodologicamente, recorre à Análise Crítica do Discurso (ADC). Caracteriza-se como uma pesquisa de cunho qualitativo e se desenvolveu analiticamente a partir da categoria Representação de Eventos (VIEIRA; RESENDE, 2016). Os resultados apontam uma representação negativa acerca do distanciamento social, que resulta em isolamento, sendo, do ponto de vista da pessoa surda e surdocega, um isolamento causado pela não interação linguística e/ou dificuldades de interação virtual devido à má qualidade da internet.
\end{abstract}

Palavras-chave: Isolamento social. Pessoa surda e surdocega. Representação discursiva.

Abstract: This work is a discussion about the experience under the measure of social distancing considering the deaf and one deafblind people perception. The main goal is to analyze the deaf and deafblind people discursive representations about measures of social distancing whose result is isolation, which happens before and/or during the Covid-19 pandemic. Theoretically, the article is based on the concept of Social Representation (MOSCOVICI, 2007) and, methodologically, it is based on the Critical Discourse Analysis (CDA). Which is characterized as a qualitative research and it is developed analytically from the Event Representation category (VIEIRA; RESENDE, 2016). The results point to a negative representation about social distancing that results in isolation, being, from the point of view of the deaf and deafblind person, an isolation caused by non-linguistic interaction and/or difficulties in virtual interaction due to the poor quality of the internet.

Keywords: Social isolation. Deaf and deafblind people. Discursive representation.

\footnotetext{
${ }^{1}$ Universidade Federal do Pará, Programa de Pós-Graduação em Cidades, Territórios e Identidades, Abaetetuba, PA, Brasil. Endereço eletrônico: lilia_olive@ hotmail.com.

${ }^{2}$ Universidade Federal do Pará, Faculdade de Ciências da Linguagem, Abaetetuba, PA; Universidade Federal de Santa Catarina, Programa de Pós-Graduação em Estudos da Tradução, Florianópolis, SC, Brasil. Endereço eletrônico: marciacarvalho369@gmail.com.

${ }^{3}$ Universidade Federal do Pará, Faculdade de Ciências da Linguagem, Programa de Pós-Graduação em Cidades, Territórios e Identidades, Abaetetuba, PA, Brasil. Endereço eletrônico: rsns@ufpa.br.
} 


\section{Introdução}

Este trabalho discorre sobre a representação discursiva do distanciamento social na

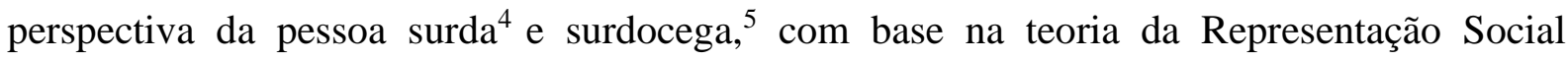
(MOSCOVICI, 2007). Segundo essa teorização, as representações sociais são assimiladas e reformuladas pelas pessoas de acordo com o contexto em que estão inseridas. Assim, o foco repousa na representação da condição de acatamento das medidas de distanciamento social. ${ }^{6}$

Em termos metodológicos, a pesquisa tem caráter qualitativo, com dados gerados por meio de entrevistas semiestruturadas com pessoas surdas e pessoa surdocega. As entrevistas ocorreram por meio de gravação de vídeos das perguntas, posteriormente encaminhados aos participantes da pesquisa por meio do aplicativo WhatsApp, pelo qual as respostas foram obtidas. Vale ressaltar que a pessoa surdocega entrevistada possui dois laudos separados: um de otorrinolaringologista e outro de oftalmologista. A surdocegueira da participante é atestada principalmente na área educacional. Pelo fato de ter perda de visão progressiva e não ser usuária da Libras tátil, foi possível realizar a entrevista a partir de vídeos em Libras.

Apresentamos transcrições, em glosa, ${ }^{7}$ das entrevistas com os participantes, bem como as análises das representações discursivas sobre as medidas de distanciamento social anterior e/ou durante a pandemia da Covid-198. Outro método mobilizado foi a Análise Crítica do Discurso (ADC), notadamente a categoria analítica Representação de Eventos (VIEIRA; RESENDE, 2016). Por fim, também utilizamos os Estudos Surdos para explanar a cultura e a comunidade surdas.

\footnotetext{
${ }^{4}$ Utilizamos o termo pessoa surda de acordo com o Art. $2^{\circ}$ do Decreto 5.626/2005: considera-se pessoa surda aquela que, por ter perda auditiva, compreende e interage com o mundo por meio de experiências visuais, manifestando sua cultura principalmente pelo uso da Língua Brasileira de Sinais - Libras.

5 "Surdo-cego - uma definição funcional se refere ao surdo-cego como aquele que tem uma perda substancial da visão e da audição, de tal modo que a combinação das suas deficiências cause extrema dificuldade na conquista de habilidades educacionais, vocacionais, de lazer e sociais. A palavra-chave nesta definição é COMUNICAÇÃO" (QUADROS, 2004, p. 10).

${ }^{6}$ Segundo o site TelessaúdeRS, da Universidade Federal do Rio Grande do Sul - UFRGS: "Distanciamento social é a diminuição de interação entre as pessoas de uma comunidade para diminuir a velocidade de transmissão do vírus. É uma estratégia importante quando há indivíduos já infectados, mas ainda assintomáticos ou oligossintomáticos, que não se sabem portadores da doença e não estão em isolamento. Esta medida deve ser aplicada especialmente em locais onde existe transmissão comunitária, como é o caso do Brasil, quando a ligação entre os casos já não pode ser rastreada e o isolamento das pessoas expostas é insuficiente para frear a transmissão. O distanciamento social pode ser ampliado (não se limita a grupos específicos) ou seletivo (apenas os grupos de maior risco ficam isolados - idosos, imunodeprimidos, pessoas com doenças crônicas descompensadas)". Disponível em: https://www.ufrgs.br/telessauders/posts_coronavirus/qual-a-diferenca-de-distanciamento-socialisolamento-e-quarentena/. Acesso em: 27 out. 2021.

${ }^{7} \mathrm{O}$ sistema de escrita por notação glosa é um tipo de transcrição de sinais, utilizado por tradutores-intérpretes de Libras, capaz de descrever frases sinalizadas em Libras, através de palavras escritas por meio de um português simplificado (BUNGEROT et al., 2008).

${ }^{8}$ A Covid-19 é uma infecção respiratória aguda causada pelo coronavírus SARS-CoV-2, potencialmente grave, de elevada transmissibilidade e de distribuição global. Disponível em: https://coronavirus.saude.gov.br/sobre-adoenca. Acesso em: 11 mai. 2021.
} 


\section{Distanciamento social e isolamento social}

A pandemia da Covid-19 provocou grandes mudanças no convívio social mundialmente. Devido o potencial de letalidade do vírus, foram necessárias medidas específicas para conter a propagação da doença. Atitudes práticas, como lavar as mãos com água e sabão, higienizá-las com álcool em gel frequentemente, usar máscaras, manter distanciamento social e fazer quarentena ${ }^{9}$ foram condições acionadas para barrar a disseminação em massa do vírus. Evitar o contato físico com outras pessoas levou à orientação de ficar em casa. Essa orientação foi, para muitas pessoas, uma condição de isolamento social ${ }^{10}$, devido à mudança brusca de rotina, como diz Lima:

O confinamento imposto pela Covid-19, que já foi descrito como o "maior experimento psicológico do mundo" (VAN HOOF, 2020), vem colocando à prova a capacidade humana de extrair sentido do sofrimento e desafiando indivíduos e sociedades, no Brasil e em todo o planeta, a promoverem formas de coesão que amorteçam o impacto de experiências-limite na vida mental (LIMA, 2020, p. 6).

O distanciamento social e/ou físico é um conjunto de ações que visa limitar o convívio social, com o propósito de controlar a propagação de doenças contagiosas. Essa medida é potencialmente eficaz, pois diminui o contato entre pessoas infectadas com outras que não foram infectadas, minimizando, assim, a transmissão da doença. Porém, precisamos olhar além do horizonte da infecção, é importante observar que:

O impacto da pandemia de coronavírus pode ser melhor apreendido não apenas através do número de infecções e mortes - por si só muito trágicos - mas olhando a forma como tocou as vidas de todos, em suas dimensões coletivas e individuais. Não há virtualmente ninguém no mundo cuja vida não tenha sido afetada, de uma maneira ou de outra, pela doença ou pelas transformações que ela impôs nas nossas vidas, sobretudo nas cidades (MEDEIROS; RAJS, 2020, p. 6).

\footnotetext{
${ }^{9}$ De acordo com a Lei $\mathrm{n}^{\circ} 13.979$ de 2020, que trata das medidas para enfrentamento da emergência de saúde pública de importância internacional decorrente do Coronavírus no Art. $2^{\circ}$ Para fins do disposto nesta Lei, considera-se: "II - quarentena: restrição de atividades ou separação de pessoas suspeitas de contaminação das pessoas que não estejam doentes, ou de bagagens, contêineres, animais, meios de transporte ou mercadorias suspeitos de contaminação, de maneira a evitar a possível contaminação ou a propagação do coronavírus". Disponível em: https://www.in.gov.br/en/web/dou/-/lei-n-13.979-de-6-de-fevereiro-de-2020-242078735. Acesso em: 27 out. 2021.

10 De acordo com o site TelessaúdeRS: "Isolamento é uma medida que visa separar as pessoas doentes (sintomáticos respiratórios, casos suspeitos ou confirmados de infecção por coronavírus) das não doentes, para evitar a propagação do vírus. O isolamento pode ocorrer em domicílio ou em ambiente hospitalar, conforme o estado clínico da pessoa. Essa ação pode ser prescrita por médico ou agente de vigilância epidemiológica e tem prazo mínimo de 10 dias do início dos sintomas e 24 horas de recuperação da doença (ausência de febre sem uso de antitérmicos e melhora dos demais sintomas), ou, para casos graves ou em pessoas gravemente imunocomprometidas, esse período pode chegar a 20 dias". Disponível em: https://www.ufrgs.br/telessauders/posts_coronavirus/qual-a-diferenca-de-distanciamento-social-isolamento-equarentena/. Acesso em: 27 out. 2021.
} 
Com a adoção das medidas de distanciamento social, a sociedade está vivenciando um pouco da sensação de isolamento já há muito tempo experienciada por pessoas com deficiência e pessoas surdas:

\begin{abstract}
A impossibilidade de convivência com seus pares e acesso aos espaços sociais tem ocasionado agravos à saúde mental da população, conforme amplamente relatado em mídias diversas. Esse isolamento representa para nós, pessoas sem Deficiência (PsD), uma exceção, um estado transitório. Entretanto, o desespero que atualmente assola as PsD espelha a vida inteira das pessoas com Deficiência, cuja participação na sociedade limita-se por obstáculos de naturezas diversas (atitudinais, arquitetônicas, cognitivas, idiomáticas, digitais, etc) (COSTA, 2020, p. 10).
\end{abstract}

A estrutura social há muito tempo limita as pessoas da participação social de acordo com suas condições. Os motivos pelos quais se chega a uma condição de isolamento são vários e, falando especificamente da perspectiva social, os grupos minoritários, que não se enquadram na condição hegemônica, são os mais afetados quanto à acessibilidade a serviços, participação ativa na dinâmica social, entre outros.

As pessoas com deficiência são historicamente atingidas por isolamentos. Elas foram consideradas, ao longo do tempo, como "um peso morto" (REIS; GATI; MACHADO, 2019), pois em virtude de sua exclusão do mercado profissional, "não dão retorno financeiro à sociedade". Muitas dessas pessoas foram rejeitadas por suas famílias e ainda hoje são recebidas com luto, porque o meio social não é favorável ao desenvolvimento delas.

As representações da deficiência sempre foram negativas, o que causa exclusão, isolamento e solidão, como diz Costa:

\begin{abstract}
A suposição de que a PcD não é capaz ocasiona danos de ordens diversas. Vale notar o custo emocional de crescer com pessoas à sua volta seguras de que você tem pouco, ou nada, a contribuir; pessoas que resumem sua existência à deficiência e sentenciam sua vida à clausura (inclusive porque suas características sensoriais, motoras, físicas e cognitivas não foram contempladas nos espaços sociais) (COSTA, 2020, p. 10).
\end{abstract}

Se entendemos, como Cavalieri e Costa (2011, p. 496), que "os modos desiguais de segregação produzem diferentes formas de vida social", podemos compreender que a forma de tratamento dedicada pela sociedade às pessoas surdas e surdocegas gerou diferentes percepções do sujeito na vida social, ou seja, as formas como elas se representam e são representadas pelos outros difere. Ainda hoje, o histórico de exclusão que acompanha as pessoas surdas e surdocegas reverbera na convivência social entre surdas, surdos, surdocegas e ouvintes. ${ }^{11} \mathrm{~A}$

\footnotetext{
11 “Ouvinte é a pessoa não surda. O termo pode simplesmente se referir a todos os que não são surdos, mas também pode ter uma conotação de não pertencimento a comunidade surda no sentido de oposição. Muitas vezes, a palavra "ouvinte" pode ser usada para se referir a quem está advogando contra os surdos ou aqueles que não compreendem a perspectiva surda. Por outro lado, quando é usada simplesmente como não ser surdo, pode se referir a qualquer pessoa que ouve e fala uma língua. Os surdos sempre se referem ao estado de ser ou não ser ouvinte: Lá naquela
} 
condição surda é vista como empecilho a uma vida socialmente igualitária, pois permanece o olhar diferenciador negativo, pejorativo e incapacitante.

Pessoas surdas resistiram e resistem à indução de estar à parte da convivência social, sempre se reuniram para sanar necessidades sociais e de linguagem. Encontrar os pares é um ato de sociabilidade frequente entre as pessoas surdas. Strobel (2009) considera os encontros de pessoas surdas (seja em rodas de conversa, reuniões descontraídas em suas residências ou em locais públicos) como um artefato cultural da comunidade surda.

Porém, a pandemia da Covid-19 impactou sobremaneira essa socialização, pois, com o perigo de contaminação pelo vírus, foram suspensos os encontros presenciais. As pessoas surdas encontraram-se em um novo isolamento, porque além de impedidas de estar com seus pares, elas tinham de estar junto a seus familiares, mas sem comunicação, uma vez que a maioria não é falante de Libras. ${ }^{12}$ Nesse sentido, Strobel $(2009$, p. 55) aponta que o isolamento dentro de casa se dá pelas barreiras de comunicação e que o fato de estar em meio às pessoas fisicamente, mas sem interação comunicativa, é caracterizado como "isolamento mental".

Na seção seguinte, apresentaremos o enquadre teórico em que a pesquisa se sustenta.

\section{A representação em Moscovici: uma reflexão sobre o diferente dentro do senso comum}

Serge Moscovici foi um psicólogo social romeno radicado na França. Constituiu a teoria da Representação Social, que surgiu com aporte na teoria da Representação Coletiva de Durkheim, o qual considerava a sociedade uma estrutura uniforme. Moscovici, entretanto, concebe a sociedade como uma estrutura multiforme, pois cada indivíduo absorve o que está a sua volta e, ao mesmo tempo, oferece uma contribuição única e peculiar. Assim, o autor assume que a sociedade é composta por representações sociais abrangentes, que se sobrepõem a todos os indivíduos e estes moldam-na de acordo com a experiência adquirida ao longo da vida, criando algo que lhes é próprio, único, mas não livre de influências do senso comum.

O contexto dos ouvintes em relação ao grupo surdo é opressor. Os dois grupos estão sempre em contato, pois são seres sociais que, de alguma forma, precisam interagir em contextos culturais e sociais. "Essa interação, na maioria das vezes, é insatisfatória pelo fato de o ouvinte não saber como se comunicar com os surdos. Esse grande problema tem resultado na exclusão e, consequentemente, gera a opressão sobre os surdos” (SANTOS JUNIOR, 2020, p.

escola só tem ouvintes. Você é ouvinte ou surdo? Você é ouvinte, como aprendeu a língua de sinais? Eu sou surdo." (QUADROS, 2019, p. 34).

12 A lei 10.436 de 2002 no Parágrafo único. Entende-se como Língua Brasileira de Sinais - Libras a forma de comunicação e expressão, em que o sistema linguístico de natureza visual-motora, com estrutura gramatical própria, constitui um sistema linguístico de transmissão de ideias e fatos, oriundos de comunidades de pessoas surdas do Brasil. 
6). Usando os termos de Moscovici (2007), assumimos que a surdez enquanto perda auditiva é parte do "universo reificado", diz respeito ao conhecimento clínico acerca da não percepção do som. Porém, no "universo consensual", a sociedade, em suas práticas cotidianas, se apropria desse dado clínico e cria representações sociais negativas e reducionistas.

De acordo com Strobel (2009), uma simples mudança de palavra que identifique uma pessoa ou um grupo pode retirar uma carga negativa ou potencializá-la. Acredita-se que é importante pensar na escolha adequada da palavra que irá representar e/ou identificar um determinado grupo, essa escolha deve servir para fazer dessas pessoas as protagonistas de sua própria história, ressaltando sempre o que podem fazer e nunca aquilo que não podem. Wilcox e Wilcox (2005, p. 17) comentam sobre a proposição de Ben Bahan ao chamar as pessoas surdas de "pessoas visuais", por envolver suas identificações socioculturais, como, por exemplo, o uso de tecnologias assistivas e a emergência da língua visual.

De acordo com os autores Wilcox e Wilcox (2005), é evidente que a plena mudança de sentido e as novas perspectivas surgidas do desejo de uma pessoa em ser identificada por sua potencialidade, e não pela falta de uma delas, gera representações sociais que favorecem as ações coletivas e individuais, em detrimento das práticas excludentes disseminadas, como pode ser visto no decurso da história e ainda é praticado atualmente. Mas, para isso, é preciso que a sociedade interaja entre si para produzir conhecimento. Moscovici (2007, p. 9), acerca da perspectiva da psicologia social, afirma que:

\begin{abstract}
O conhecimento nunca é uma simples descrição ou uma cópia do estado de coisas. Ao contrário, o conhecimento é sempre produzido através da interação e comunicação e sua expressão está sempre ligada aos interesses humanos que estão nele implicados. $\mathrm{O}$ conhecimento emerge do mundo onde as pessoas se encontram e interagem, do mundo onde os interesses humanos, necessidades e desejos encontram expressão, satisfação ou frustração. Em síntese, o conhecimento surge das paixões humanas e, como tal, nunca é desinteressado; ao contrário, ele é sempre produto dum grupo específico de pessoas que se encontram em circunstâncias específicas, nas quais elas estão engajadas em projetos definidos [...] Uma psicologia social do conhecimento está interessada nos processos através dos quais o conhecimento é gerado, transformado e projetado no mundo social (MOSCOVICI, 2007, p. 9).
\end{abstract}

Logo, se as representações sociais sobre pessoas surdas fossem positivas, sua qualidade de vida seria completamente diferente, e o isolamento social e mental em que elas vivem neste contexto pandêmico seria tal qual ao de ouvintes. Para haver representação social é necessário que informações majoritárias, e, portanto, dominantes, estejam em diferentes âmbitos do cotidiano. Nessa ótica, a parcela ouvinte é favorecida por ser majoritária, por ser o modelo previsto no senso comum, o "normal", o aceitável pela maioria, a ponto de a estrutura social 
ser arquitetada aos moldes dos ouvintes, o que causa impacto psicológico negativo nas pessoas surdas. Para Carvalho (2020, p. 58), é preciso mirar essa minoria:

\begin{abstract}
Pensar em surdos é pensar em minoria, que nos remete ao entendimento de que há uma maioria dominante - os ouvintes que estão em vantagem com relação aos surdos no quesito à participação pública e acesso à linguagem. Essa luta por poder e espaço envolve a questão de acesso ao discurso que afeta diretamente o ser humano. Diante disso, o acesso à língua, às mídias sociais, à informação é importante para que qualquer indivíduo possa se posicionar e se firmar socialmente (CARVALHO, 2020, p. 58).
\end{abstract}

Por isso, é necessário que a sociedade perceba e reformule suas representações sociais, promovendo engajamento tanto para a experiência visual como para a experiência sonora. Para que isso aconteça, é preciso que a comunidade surda seja vista, que essa questão seja pautada nas redes sociais, na educação etc. É urgente educar a sociedade acerca do estilo de vida de pessoas surdas, sua cultura e sua língua, para que todos compreendamos que é natural, como o estilo de vida da pessoa ouvinte também o é.

Experiência visual significa a utilização da visão, em (substituição total a audição), como meio de comunicação. Desta experiência visual surge a cultura surda representada pela língua de sinais, pelo modo diferente de ser, de se expressar, de conhecer o mundo, de entrar nas artes, no conhecimento científico e acadêmico. A cultura surda comporta a língua de sinais, a necessidade do intérprete, de tecnologia de leitura (PERLIN; MIRANDA, 2003, p. 218).

Por essa razão, a interação é urgente, assim como o desejo de paridade com a experiência de percepção do mundo visual e ouvinte, pois elas promoverão o surgimento de novas representações e darão um novo estilo de convivência harmônica entre os dois grupos. Moscovici (2007) endossa a participação ativa do indivíduo na construção dos objetos representados, é informação assimilada e refletida, depois transformada em algo que lhe é próprio. Por esse motivo, é importante que as representações sociais existentes sejam completamente modificadas para que surjam outras sem a carga negativa atual, representações que foram construídas por décadas, em contextos históricos de desvalorização da pessoa surda. Daí serem necessárias outras formas de representar e de identificar, como propõe Patrocínio:

Pensar narrativas autobiográficas produzidas por sujeitos surdos é estar diante de relatos formados a partir de uma experiência ontológica intrinsecamente ligada à materialidade de um corpo. A história nos revela as muitas interdições sofridas por esse corpo. Seja pelas inúmeras tentativas de cerceamento e proibição do uso de uma língua gesto-visual ou pelo seu completo isolamento (PATROCÍNIO, 2020, p. 91).

Sempre haverá relações de poder envolvendo diferentes grupos sociais, bem como mercado de produção e consumo de bens materiais e imateriais que manipulam a dinâmica do trabalho em sociedade e estilos de vida, mobilizados pela indústria cultural pautada no 
consumo. Sabemos que existem pessoas surdas que ainda estão caminhando apartadas, em busca de entrosamento, reconhecimento e valorização da sua mão de obra, do poder de consumo, da cultura, de estilo de vida, de espaços etc., pois ainda está enraizada a representação social da invalidez da pessoa surda, que se reflete nas formas como elas são tratadas em diversos contextos. Dependendo de como a sociedade concebe a surdez, ela é responsável pelo reflexo das configurações discursivas sobre quem é surda ou surdo:

\begin{abstract}
Em outras palavras, a surdez é lida enquanto reflexo e efeito primeiro de configurações discursivas de saber-poder que resultam em representações que transitam entre um modelo médico terapêutico - baseado em uma perspectiva que nomeia o sujeito surdo enquanto deficiente e lança mão de estratégias e recursos clínicos que almejam a sua reabilitação - e um modelo socioantropológico - fundado em uma perspectiva culturalista inspirada nos Estudos Culturais que nomeia a surdez enquanto uma diferença étnico-linguista que constitui uma cultura própria, a Cultura Surda (PATROCÍNIO, 2020, p. 92).
\end{abstract}

Na sociedade, majoritariamente ouvinte e com baixa adesão de políticas públicas que viabilizem a acessibilidade de pessoas surdas, é natural que predomine no imaginário familiar, com raras exceções, o modelo clínico-terapêutico, em detrimento à visão socioantropológica da surdez. No contexto de distanciamento social, em função da Covid-19, acredita-se que houve aumento do reflexo negativo do isolamento social da pessoa surda, que já é lida socialmente como alguém isolado na interação linguística e noutras situações do cotidiano. Na próxima seção, apresentaremos os métodos de geração e análise de dados.

\title{
Procedimentos metodológicos
}

Esta é uma pesquisa qualitativa, pois se alinha a conjuntos de práticas materiais e interpretativas que permitem transformar aspectos do mundo em representações, por meio das quais se pode entendê-los, descrevê-los e interpretá-los (DENZIN; LINCOLN, 2006). Para obter os dados da pesquisa, utilizou-se a entrevista semiestruturada, realizada virtualmente com quatro participantes por meio da Libras. As informações estão dispostas no quadro 1.

Quadro 1 - Dados das(os) participantes da entrevista

\begin{tabular}{|c|l|l|l|l|l|}
\hline Participante $^{13}$ & Sexo & Idade & Diagnóstico & Língua & Escolaridade \\
\hline Pessoa $A$ & feminino & 20 anos & Surdocega de nascença & Libras & Ensino Médio completo \\
\hline Pessoa $B$ & masculino & 20 anos & Surdo de nascença & Libras & Cursa Pedagogia \\
\hline Pessoa $C$ & feminino & 40 anos & Surdez adquirida aos 10 anos & Libras & Ensino Médio completo \\
\hline Pessoa $D$ & feminino & 19 anos & Surda de nascença & Libras & Ensino Médio completo \\
\hline
\end{tabular}

Fonte: elaborado pelas autoras (2021).

${ }^{13}$ A fim de preservar os nomes reais das pessoas participantes, utiliza-se o termo Pessoa seguido de letra do alfabeto. 
A participante Pessoa A informou que a sua constituição familiar é de pessoas ouvintes (mãe e padrasto), sendo ela surdocega e filha única. Além disso, afirmou que sua mãe é professora na área da educação especial, militante na causa das pessoas surdas e usuária da Libras. O participante Pessoa $B$ é um jovem surdo de nascença, universitário, pertence a uma família de ouvintes na qual os membros sabem Libras. A Pessoa $C$ é casada com um ouvinte e mãe de uma pessoa ouvinte. Atualmente, trabalha com venda de comidas, mas foi auxiliar administrativa em uma empresa da região. Foi ouvinte e perdeu a audição quando criança devido a complicações num quadro de meningite. Além de saber Libras e português escrito, é oralizada. A pessoa $D$ faz um pouco de leitura labial e alguns membros da família sabem Libras. Ela ministra aula de Libras para crianças em um curso livre no município de Abaetetuba, no Estado do Pará. Todas as entrevistas foram realizadas em Libras, o que demandou transcrição das respostas em forma de glosa, em função da modalidade visual da língua.

Para a análise dos dados, utilizou-se a Representação de Eventos, categoria de análise textual da ADC. Em resumo, essa categoria analítica está diretamente relacionada ao significado representacional e a discursos particulares. As questões pertinentes para análise nessa categoria estão de acordo com os questionamentos de Vieira e Resende (2016, p. 117): "Que elementos dos eventos sociais representados são incluídos ou excluídos? Que elementos incluídos são mais salientes? Quão abstrata ou concretamente os eventos são representados?”.

\footnotetext{
O discurso figura na representação do mundo material, de outras práticas sociais ou em representações autorreflexivas da própria prática particular. Essas representações particulares de aspectos do mundo se realizam discursivamente e variam conforme as diferentes perspectivas ou posições dos sujeitos nas práticas sociais. Discursos particulares constituem, então, modos particulares de representar a realidade, por isso um dos significados do discurso é o representacional, ainda que sempre mantenha relações dialéticas com os outros significados. Relaciona-se ao eixo do saber de Foucault (1994) (VIEIRA; RESENDE, 2016, p. 179).
}

Toda essa reflexão também está apoiada no conceito teórico de Representações Sociais, de Moscovici (2007). Nesta pesquisa, focamos no significado representacional e a análise se refere ao recorte de duas perguntas feitas nas entrevistas. A primeira aborda a percepção sobre o coletivo e a segunda aborda a experiência pessoal: 1. Sabemos que o momento pandêmico é complexo e provocou mudanças no convívio social, mas como você percebe o grupo surdo no isolamento social? 2. Devido às medidas de proteção os encontros em grupos de forma presencial foram evitados e substituídos por encontros virtuais, como você se sente? Como você percebe esse momento de isolamento social? Na seção seguinte, apresenta-se os trechos da entrevista com as (os) participantes, destacando as expressões que fazem referência às representações sobre o distanciamento social, destacados na glosa. 


\section{Análise das entrevistas}

As análises seguem a ordem do quadro 1. Primeiro mostramos os excertos (transcrição em glosa) e as análises em seguida. O trecho abaixo é da entrevista com a Pessoa A.

Quadro 2 - Transcrição da entrevista com a Pessoa A

\section{Glosa}

SEMPRE SAÚDE CONTINUAR FICAR EM CASA AQUI! (não tem jeito) AQUI JÁ.

FICAR SOZINHO PRECISA MÁSCARA, PRECISA MÁSCARA SAIR COMPRAR COMIDA, RETORNAR CASA, GUARDAR MÁSCARA, TODOS FICAR SOZINHO, PORQUE TEMPO ATRÁS MINHA MÃE FOI HOSPITAL FAZER FURAR DEDO, SANGUE, NADA, RUIM NADA, ENTENDEU? $[\ldots]$

[...] SIM, SIM, SIM MULHER FAMÍLIA SOZINHA NÃO TER LIBRAS NÃO TER, AQUI SÓ MÃE, APRENDER TI@, SÓ. NÃO TER FAMÍLIA NÃO TEM, POXA (expressão de insatisfação) COMO?! (mão bate na testa com expressão de desapontamento, porém risonha) EU NÃO TER TODOS CASA FAMÍLIA, NÃO TER, MAMÃE NÃO TER, TIA SOZINHA NÃO TER FAMÍLIA, FICAR CALADA E ACABAR (expressão de indiferença) [...]

EU SENTIR SÓ NÃO, NORMAL, SORRISO, FELIZ, RESPEITO/OBEDECER SÓ, MAS MEU PAI BELÉM BEBER (bebida alcoólica) MUITO, EU PREOCUPADA, SÓ PREOCUPAÇÃO. VER MEU PAI TONTO, ENTENDE? SÓ.

EU CALADA SURDA VOU FELIZ (cabeça erguida) SÓ OBSERVANDO, (nariz empinado). [...] MAMÃE AMIGA, MAS ELA FICA MUITO COMPUTADOR, OLHA (mão bate na testa). ENTÃO, SOZINHA. VOCÊ FELIZ É? (expressão de estar analisando) (vídeo de 02:02s)

Fonte: elaborado pelas autoras (2021).

No excerto acima, é possível perceber que a Pessoa $A$ se mostra consciente quanto à necessidade de ficar em casa e de usar máscara para se proteger da contaminação pelo coronavírus, como mostra o trecho "FICAR SOZINHO PRECISA MÁSCARA, PRECISA MÁSCARA SAIR COMPRAR COMIDA, RETORNAR CASA, GUARDAR MÁSCARA”. Também sabe que essa condição é para toda a sociedade, pois afirma que "TODOS FICAR SOZINHO". Porém, revela haver uma barreira comunicacional em sua casa, tendo em vista que apenas sua mãe sabe Libras: “MULHER FAMÍLIA SOZINHA NÃO TER LIBRAS NÃO TER, AQUI SÓ MÃE, APRENDER TI@, só". Nesse sentido, a interação se dá apenas com a mãe, que já sabe Libras, e com a tia, que está em fase de aprendizagem da língua.

$\mathrm{O}$ primeiro isolamento a que a Pessoa $A$ se refere diz respeito às recomendações de distanciamento social, à impossibilidade de ela estar em contato com pessoas falantes de Libras, com as quais possa socializar de forma efetiva. Em "FICAR CALADA E ACABAR", a Pessoa A reitera essa dificuldade de comunicação, o que indica um isolamento dentro do confinamento domiciliar. Concordamos com Strobel (2009) quando ela afirma que o isolamento no ambiente doméstico ocorre pelas barreiras de comunicação, não é suficiente estar no meio das pessoas fisicamente, pois a falta de interação comunicativa resulta em "isolamento mental".

Com Costa (2020) compreendemos que o isolamento é duplamente difícil a pessoas surdas e com deficiência. Primeiro porque é necessário atender às exigências das medidas de segurança, o que inviabiliza a convivência com os pares e o acesso aos espaços sociais. Segundo 
porque essas pessoas já sofrem com a exclusão social, em grande parte de suas vidas, por imporlhes "obstáculos de naturezas diversas (atitudinais, arquitetônicas, cognitivas, idiomáticas, digitais, etc.) (COSTA, 2020, p. 10).

Além disso, o uso do termo normal em "EU SENTIR SÓ NÃO, NORMAL, SORRISO, FELIZ, RESPEITO/OBEDECER SÓ” aponta que a Pessoa A normalizou a condição de estar sozinha. Como já disse Moscovici (2007), cada indivíduo absorve o que está a sua volta e, ao mesmo tempo, oferece sua contribuição peculiar, moldando a sociedade de acordo com a experiência adquirida ao longo de sua vida. Tal representação da solidão é reiterada no trecho "MAMÃE AMIGA, MAS ELA FICA MUITO COMPUTADOR, OLHA (mão bate na testa). ENTÃO, SOZINHA", em que a participante fala sobre o trabalho remoto de sua mãe neste tempo, apontando-o como um complicador, já que interfere na interação comunicativa no ambiente familiar. Assim, as medidas de distanciamento social em função da pandemia da Covid-19 agravaram a falta de interação, tanto no seio familiar quanto em outros ambientes.

O próximo excerto é da entrevista com a Pessoa B. Vejamos qual sua percepção desse período de distanciamento no quadro 3:

Quadro 3 - Transcrição da entrevista com a Pessoa B

EU ACHO GRUPO SURDO ALGUNS ISOLAMENTO SURDO ESPERAR DEMORA, MAS MÃE ME AJUDA ME PROTEGER, PAPAI ME AJUDA PROTEGER TAMBÉM, FAMÍLIA CONVERSAR AJUDA SEMPRE. ENTÃO, SÓ WHATSAPP, MENSAGEM, VÍDEO CHAMADA (web), CONVERSAR. EU ACHO PORQUE SURDO, ALGUNS, INTERNET DEFEITO, IMAGEM EMBAÇADA, ENTENDEU? SÓ. EU ESPERO DEMORA, EU PERCEBO ALGUMAS PESSOAS SURDAS CASA FICAR SEMPRE DESPREZO, DEIXA PRA LÁ, FAZ POUCO CASO, SAIR PASSEAR, ACONTECE PORQUE CORONA PESSOA PEGA, DOENÇA PERIGOSA, CUIDADO SIM, ENTÃO CALMA PREOCUPAÇÃO, CALMA, OLHAR/OBSERVAR, ESPERAR TEMPO PASSAR, ANO NOVO, OBSERVAR JANEIRO MÉDICO PESQUISA VACINA CONSEGUIR ACABAR JANEIRO, FEVEREIRO OU MARÇO. FICAR OBSERVANDO TELEVISÃO, ACABOU CORONA? OK, SAIR, PASSEAR, SAUDADE ENCONTRAR TODOS, ENTENDEU? (vídeo de 01:42s)

SEGUNDO PONTO, SURDO ESTUDAR COMO? PORQUE LIVE INTERNET NÃO CONSEGUE PORQUE CAI, DEFEITO, IMAGEM RUIM, DIFÍCIL, CASA FICAR SEMPRE, ANO DEMORA SEGUIR, SÓ UFPA, TAMBÉM UEPA, FACULDADE ESTUDAR TER INTERNET, INTERNET NÃO SÓ WIFI, ALGUNS AMIGOS VISITA, CONTATO JUNTO AMIGO, LIVE, ENTENDEU? EU ACHO. PORQUE ALGUNS ALUNOS FACULDADE ESTUDA OUVINTE LÁ INTERNET NÃO CONSEGUE, DEFEITO, IMAGEM EMBAÇADA, NÃO CONSEGUE. SÓ FACULDADE ENTRAR SÓ NÚMERO 6, JUNTO NÃO, SENTADOS LONGE, SEPARADO, PROFESSOR ENSINA, PERÍODO ATÉ DIA 17 FIM, ACABOU, LEGAL, ACABOU, BOM, SÓ GRUPO INTERNET. EU GRUPO FACULDADE ESTUDAR. EU COMEÇAR SÓ WIFI, LIVE SEMPRE, ENTENDEU? EU PENSO SURDO ALGUNS ESTUDAR CASA ESPERAR LIVE NÃO TER NADA, NÃO CONSEGUE NADA, EU ACHO, NÃO SEI, TALVEZ, ENTENDEU? (vídeo de $01: 21 s)$

Fonte: elaborado pelas autoras (2021).

A Pessoa $B$ não se sente isolada, pois não há barreira comunicativa como aquela experienciada pela Pessoa A. No fragmento "FAMÍLIA CONVERSAR AJUDA SEMPRE", podemos observar que o isolamento não está relacionado à impossibilidade de interação comunicativa. Embora nessa família a pessoa surda não se considere isolada, porque consegue se comunicar, 
o prejuízo está na falta de comunicação noutros âmbitos, como o educacional, por exemplo: “SEGUNDO PONTO, SURDO ESTUDAR COMO? PORQUE LIVE INTERNET NÃO CONSEGUE PORQUE CAI, DEFEITO, IMAGEM RUIM, DIFÍCIL, CASA FICAR SEMPRE”. Desse modo, por mais que haja interação, na maioria das vezes, ela não é satisfatória, resultando na exclusão, que gera opressão a pessoas surdas (SANTOS JUNIOR, 2020).

Ela representa negativamente esse isolamento quando comenta sobre outros surdos que sofrem desprezo no ambiente familiar em "PERCEBO ALGUMAS PESSOAS SURDAS CASA FICAR SEMPRE DESPREZO, DEIXA PRA LÁ, FAZ POUCO CASO”. A necessidade de estar com os pares leva-os a quebrar o distanciamento social, como indica este trecho: "SAIR PASSEAR, ACONTECE PORQUE CORONA PESSOA PEGA, DOENÇA PERIGOSA". Como consequência da falta de interação no ambiente familiar, essas pessoas surdas se arriscam em busca de contextos mais propícios a trocas interativas, a fim de diminuir a sensação de isolamento.

Ademais, a Pessoa $B$ lança luz sobre as precárias condições que limitam a interação virtual. Segundo ele, "INTERNET NÃO CONSEGUE PORQUE CAI, DEFEITO, IMAGEM RUIM, DIFÍCIL”, um complicador em emergência durante o distanciamento, pois, embora sejam uma ferramenta eficiente para minimizar a solidão das pessoas surdas, as tecnologias digitais apresentam obstáculos relacionados à qualidade dessa acessibilidade. Sobre isso, Perlin e Miranda consideram que "a cultura surda comporta a língua de sinais, a necessidade do intérprete, de tecnologia de leitura (2003, p. 218). As tecnologias surgem como possibilidade de manter atividades escolares, o que poderia amenizar a sensação de isolamento social, mas as condições reais se apresentam limitadas.

Conforme Carvalho (2020), é preciso dar atenção à questão da acessibilidade tecnológica para comunidade surda. Embora haja diferenças no interior mesmo da comunidade, isso torna-se mais evidente quando a comparamos com a comunidade ouvinte. Esta, por sua vez, tem mais acesso aos meios de comunicação que a comunidade surda, o que coloca a comunidade surda em posição de desvantagem nos quesitos participação pública e acesso à linguagem. Isso impõe luta por poder e espaço, que afeta diretamente o ser humano. Para a autora (2020, p. 58), é preciso garantir à comunidade surda os mesmos direitos, visto que “acesso à língua, às mídias sociais, à informação é importante para que qualquer indivíduo possa se posicionar e se firmar socialmente". Então, o acesso à informação por meio da tecnologia digital diminui, de certa forma, a angústia causada pelo distanciamento social.

No trecho “EU COMEÇAR SÓ WIFI, LIVE SEMPRE, ENTENDEU? EU PENSO SURDO ALGUNS ESTUDAR CASA ESPERAR LIVE NÃO TER NADA, NÃO CONSEGUE NADA", a Pessoa B demonstra conhecer as diferenças de acesso, apontando as questões econômicas que afetam sua 
comunidade, de modo que o distanciamento social se apresenta mais crítico àquelas pessoas que não têm acesso à internet. A comunidade surda necessita que a transmissão de vídeo funcione adequadamente nos aplicativos de interação, aspecto contornável para pessoas ouvintes, já que as câmeras podem ser desligadas a fim de tornar mais fluida a transmissão.

A seguir, no quadro 4, há um trecho da entrevista com a participante Pessoa $C$.

Quadro 4 - Transcrição da entrevista com Pessoa C

EU ACHO UM POUCO, PORQUE NO PASSADO, ANO PASSADO, CORONA NÃO TER. SURDO GOSTAR SAIR, SAIR, SAIR, SAIR, PASSEAR, RUA TODO DIA SAIR SAIR SAIR SAIR, PORQUE GOSTAR LIBRAS CONVERSAR, PAPEAR (conversas descontraídas). SURDO TAMBÉM VAI CASA OUTRO AMIGO SURDO É MUITO BOM, É LEGAL, TODOS SEMPRE CONVERSAR NORMAL. MAS, AGORA ESSE ANO MUITO DIFÍCIL PORQUE COMEÇOU CORONA, SURDO PRECISA TER MUITO CUIDADO, FICAR CASA, CUIDADO PRECISA, SE PROTEGER, MAS TAMBÉM, ALGUNS SURDOS NÃO OBEDECER PAI E MÃE, SAIR SAIR SAIR RUA SIM! ALGUNS, SÓ ALGUNS HOMENS ALGUMAS, MULHERES SURDAS SAI, SAI, SAI, MAS ÀS VEZES MÁSCARA, ÀS VEZES NÃO TER MÁSCARA. OBEDECER NADA. AGUENTAR. MAS ALGUNS FICAR CASA SIM. QUERER SE PROTEGER SAÚDE PRECISA INTERAGIR, FICAR CASA. MUITO ACONTECER MUITAS COISAS, ALGUMAS FAMÍLIAS MORRER. DIFÍCIL. TRISTE. TODO DIA SEMPRE PEDIR DEUS ABENÇOAR, PROTEGER. EU ACHO SÓ (vídeo de 02:45s).

TAMBÉM EXPLICAR, EU ESQUECI, FALTOU, MAS EU ALGUNS SURDOS ENTENDE ORALIZAR, IR COMPRAR VÁRIAS COISAS LOJA, PESSOA VEM ATENDER EU DIZER "EU SURDA", PESSOA USA MÁSCARA, EU CHAMO E DIZER "DESCULPA, EU SOU SURDA" MAS A PESSOA CONTINUA FALANDO (com máscara) EU OLHO MÁSCARA ORALIZAR ENTENDER NADA! EU DE NOVO "EI, DESCULPA EU SOU SURDA" PESSOA PARECE NÃO ENTENDER! SURDA! ALGUNS SABER ORALIZAR, ALGUNS NÃO, MAS A PESSOA SEMPRE FALA FALA FALA COM MÁSCARA DE NOVO. DIFÍCIL! SURDO NÃO ENTENDER. EU JÁ ACONTECER VÁRIAS. DE NOVO E DE NOVO, EU IR COMPRAR LOJA, SUPERMERCADO, MÉDICO. AH! MÉDICO, ELE SINAL (sinal próprio) RONNIE ELE MÉDICO EU GOSTAR IR PORQUE ELE SABE ORALIZAR, EU LEITURA LABIAL, LÁ MÁSCARA POSSÍVEL, MAS EU CHEGAR, TEMPO ATRÁS, NOVEMBRO EU ACHO NÃO LEMBRO, EU FUI LÁ. ELE MÉDICO RONNIE MÁSCARA TIRAR E FALAR. BOM! EU GOSTO DELE PORQUE ELE SABE EU PRECISO LER BOCA (LEITURA LABIAL) ELE MÁSCARA TIRA FALAR, EXPLICAR PROBLEMA, DETALHES, EU ENTENDI TUDO. ELE É ÓTIMO! MÉDICO RONNIE EU GOSTO. TAMBÉM JÁ IR LOJA VÁRIAS, MÁSCARA ALGUMAS PESSOAS RESPEITAM PORQUE SABEM EU SURDA, LÁ ALGUMAS MÁSCARA TIRA FALAR, ALGUMAS MÁSCARA NÃO TIRAR. DIFÍCIL! AGORA DIFÍCIL TODOS SURDOS ORALIZAR. OUTROS SURDOS NÃO ENTENDER ORALIZAR, SÓ LIBRAS, MAS LÁ A PESSOA LOJA NÃO SABE LIBRAS, SÓ ORALIZA, SURDO NÃO ENTENDER NADINHA. MUITO DIFÍCIL. EU COMPRO VÁRIAS COISAS DE FESTA LÁ LOJA DE COISAS FESTA VÁRIAS, LÁ TER UMA MULHER OUVINTE SABE LIBRAS. EU CHEGAR LÁ COMPRAR "DESCULPA, EU SURDA" MULHER "AH TA! ESPERAR" ELA CHAMAR OUTRA MULHER SABE LIBRAS. LEGAL, EU ALEGRE! GOSTAR MUITO, EMOÇÃO, PORQUE ELA SABE LIBRAS ME EXPLICOU BEM. ALGUMAS OUTRAS LOJAS TER INTÉRPRETE SABER, TER. É BOM! SEMPRE EM CADA UMA LOJA, SUPERMERCADO, MÉDICO, QUALQUER LUGAR PRECISA TER INTÉRPRETE, IMPORTANTE PORQUE NÓS SURDOS ENTENDER MELHOR (vídeo de 04:29s).

Fonte: elaborado pelas autoras (2021).

A participante Pessoa $C$ compara o contexto anterior à pandemia ao contexto atual em "EU ACHO UM POUCO, PORQUE NO PASSADO, ANO PASSADO, CORONA NÃO TER. SURDO GOSTAR SAIR, SAIR, SAIR, SAIR, PASSEAR, RUA TODO DIA SAIR SAIR SAIR SAIR, PORQUE GOSTAR LIBRAS CONVERSAR, PAPEAR”. Ela informa que a vida social da pessoa surda é bastante ativa em tempos normais, embora limitada à comunidade que partilha a Libras. A fala da participante alinha-se à consideração de Strobel (2009, p. 51), de que são “os encontros de 
surdas e surdos (seja em rodas de conversa, reuniões descontraídas na residência delas ou deles, ou em locais públicos) como um artefato cultural da comunidade surda".

Ainda nesse mesmo escopo, a expressão conversar normal, em “TODOS SEMPRE CONVERSAR NORMAL", foi usada em referência ao período anterior ao distanciamento social. Para marcar o presente, a participante enuncia "MAS, AGORA ESSE ANO MUITO DIFÍCIL PORQUE COMEÇOU CORONA, SURDO PRECISA TER MUITO CUIDADO, FICAR CASA" e indica a dificuldade que alguns surdos têm em acatar o distanciamento social: "ALGUNS SURDOS NÃO OBEDECER PAI E MÃE, SAIR SAIR SAIR RUA SIM! ALGUNS, SÓ ALGUNS HOMENS ALGUMAS, MULHERES SURDAS SAI, SAI, SAI, MAS ÀS VEZES MÁSCARA, ÀS VEZES NÃO TER MÁSCARA”.

O termo aguentar, em "AGUENTAR. MAS ALGUNS FICAR CASA SIM. QUERER SE PROTEGER SAÚDE PRECISA INTERAGIR, FICAR CASA", está relacionado ao esforço de manterse distante dos eventos que antes eram comuns e à necessidade de interação, apontando o paradoxo a que a pessoa surda está submetida ao ter de equilibrar as necessidades de proteção e de interação com outras pessoas surdas.

Outra questão importante é estabelecimento de interação com o uso de máscaras quando a leitura labial é requerida. O trecho "ALGUNS SURDOS ENTENDE ORALIZAR, IR COMPRAR VÁRIAS COISAS LOJA, PESSOA VEM ATENDER EU DIZER “EU SURDA", PESSOA USA MÁSCARA, EU CHAMO E DIZER "DESCULPA, EU SOU SURDA" MAS A PESSOA CONTINUA FALANDO (com máscara) EU OLHO MÁSCARA ORALIZAR ENTENDER NADA!” se coaduna a Moscovici (2007), quando ele aponta que a surdez, enquanto perda auditiva, é parte do "universo reificado", que leva em conta o conhecimento clínico acerca da não percepção do som. A sociedade, em suas as práticas cotidianas, apropria-se desse dado clínico e cria representações sociais negativas e reducionistas das pessoas surdas, em razão de elas não se encaixarem como ouvintes. Logo, na maioria das vezes, é a pessoa surda quem tem de se adequar aos padrões de comunicação oral.

Segundo a Pessoa $C$, até a pessoa surda oralizada tem severas dificuldades de interação quando as especificidades dessa comunicação não são atendidas, como a visualização dos lábios, por exemplo. Essa dificuldade pode ser ultrapassada por meio do uso de Equipamento de Proteção Individual (EPI), como máscaras específicas que permitem a visualização da região da boca no momento da oralização. Dessa feita, a não utilização de recurso desse tipo pode tornar a comunicação inacessível, mesmo a uma pessoa surda oralizada, como é possível observar em “A PESSOA SEMPRE FALA FALA FALA COM MÁSCARA DE NOVO. DIFÍCIL!”. Alguns exemplos de máscaras acessíveis à leitura labial estão nesta imagem: 
Figura 1 - Máscaras acessíveis

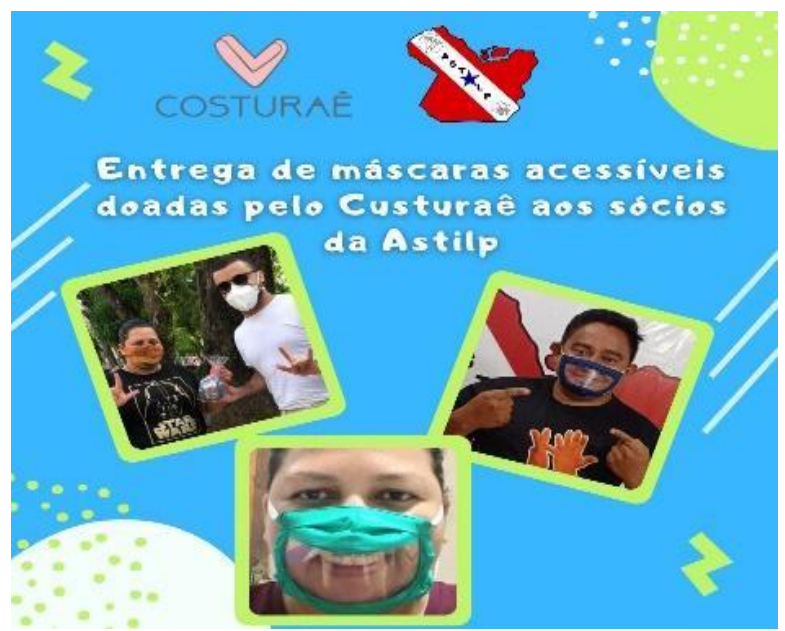

Fonte: ASTILP ${ }^{14}$.

Reconhecer as especificidades de comunicação da pessoa surda faz o acesso a serviços básicos de saúde mais eficiente, seja em contexto de distanciamento social ou não. Por exemplo, a Pessoa $C$ narra sua boa experiência em consulta médica assim: “AH! MÉDICO, ELE SINAL (sinal próprio) RONNIE ELE MÉDICO EU GOSTAR IR PORQUE ELE SABE ORALIZAR, EU LEITURA LABIAL, LÁ MÁSCARA POSSÍVEL, MAS EU CHEGAR, TEMPO ATRÁS, NOVEMBRO EU ACHO NÃO LEMBRO, EU FUI LÁ. ELE MÉDICO RONNIE MÁSCARA TIRAR E FALAR. BOM! EU GOSTO DELE PORQUE ELE SABE EU PRECISO LER BOCA (LEITURA LABIAL) ELE MÁSCARA TIRA FALAR, EXPLICAR PROBLEMA, DETALHES, EU ENTENDI TUDO. ELE É ÓTIMO!”. Nesse caso, a Pessoa $C$ evidencia que a comunicação entre a pessoa surda e a pessoa ouvinte pode ser atingida de modo satisfatório quando a comunidade ouvinte conhece as particularidades envolvidas na interação com a pessoa surda. Em que pese o risco de contaminação, "tirar a máscara" foi tomada como opção viável a fim de haver troca de informação. Esse risco já pode ser evitado com a utilização de máscaras de acessíveis, como ilustramos por meio da figura 1.

Por fim, no fragmento "SEMPRE EM CADA UMA LOJA, SUPERMERCADO, MÉDICO, QUALQUER LUGAR PRECISA TER INTÉRPRETE, IMPORTANTE PORQUE NÓS SURDOS ENTENDER MELHOR", a participante destaca a necessidade de haver intérpretes nos espaços sociais para garantir acessibilidade linguística. Em sua entrevista, ela não se limitou a comentar apenas o distanciamento em função da pandemia, mas também disse de seus anseios de maneira geral.

A seguir, apresentamos a transcrição da entrevista com a Pessoa D.

\footnotetext{
14 Associação de tradutores intérpretes de Língua de Sinais do Pará. O Custuraê é um projeto da Enactus (organização internacional sem fins lucrativos dedicada a inspirar discentes a melhorar o mundo através da Ação Empreendedora). Mais informações em: http://www.enactus.org.br/. Informações sobre a pandemia e sobre o projeto em: https://youtu.be/sApBtgd9s8w- I- UFPA Enactus- 2021. Acesso em: 15 jun. 2021.
} 
Quadro 5 - Transcrição da entrevista com a Pessoa D

[...] EU AQUI CASA, PASSEIO UM POUQUINHO, UM POUQUINHO, MAIS OU MENOS PORQUE AQUI CASA SENTIR RUIM, LEGAL NÃO, ENTÃO EU SENTIR RUIM, MAS TODO DIA NÃO, DIA PASSEIO SEGUNDA, QUARTA MAIS OU MENOS. CONVIDO IR JUNTO PASSEAR, QUANDO VOLTAR PRECISA BANHO, SABÃO, SAÚDE. PRECISA MÁSCARA ME PROTEGER SE VISITAR AMIG@ OUTRA CASA [...]

ENTÃO, FAMÍLIA MINHA, DESCULPA, OUTRAS FAMÍLIAS NÃO TER LIBRAS, SOZINH@, AINDA NÃO COMUNICAÇÃO, SENTIR SOZINHO, DESEJAR PENSAR AMIGO SURDO CONVIDAR IR JUNTO NÓS DUAS IR TOMAR SORVETE, CONVERSAR, VOLTAR JÁ CONVERSAR UM POUQUINHO, MAS PROBLEMA NÃO TER, EU VEJO PESSOAS ANDANDO NA RUA, SINTO CALMA E SÓ, ENTENDEU? [...]

EU FICO OBSERVANDO O FACEBOOK, VÍDEOS, SURDO FAMÍLIA GRUPO SABE LIBRAS PAI, MÃE SURDO, CRIANÇAS SURDAS TAMBÉM COMUNICAÇÃO, EU VEJO EU SINTO EMOÇÃO, OBSERVAR O APRENDIZADO, ENTENDEU? EU GOSTO, ACHO MUITO LEGAL. AINDA PESSOAS AQUI NÃO TEM. OBSERVANDO VÁRIAS PESSOAS NÃO TEM AQUI EU ESPERANDO SOZINHA TRABALHAR AQUI DENTRO (expressão de tédio/chato), PACIÊNCIA [...]

DIFÍCIL. ÀS VEZES EM CASA SURDO SOZINHO SENTIR OUTROS PORQUE PARECE PESSOAS MENOSPREZAR, NÃO QUERER PERTO ENTÃO SOZINHO. PRECISA ESPERANÇA, UNIÃO NOSSA FAMÍLIA. DIFERENTE PESSOAS OUVINTES, SAI, VOLTA, PASSEIA, CASA DENTRO PODE PEGAR DOENÇA, PERIGOSO. O SURDO PRECISA RESPEITO SAIR NÃO, PRECISA RESPEITAR, ESPERAR [...] SIM, SIM, SOFRIDO [...]

ENTÃO, EXEMPLO, FESTA AMIG@ (pausa com expressão de tristeza) DIFÍCIL, MAS JÁ ACONTECEU FAMÍLIA MINHA EU SOZINHA, FAMÍLIA INTERAGINDO CONVERSANDO ORALMENTE E EU CHAMAR MINHA IRMÃ PERGUNTAR "O QUE É??", MAS ELA “VAI SÓ DEFICIENTE LIBRAS SÓ”, VOLTAR CONVERSAR ORALMENTE. EU AGUENTAR (expressão de tristeza), NÃO SINTO ALEGRIA. EU SINTO (barreira). MAS ESPERANÇA, AGUENTAR, UM DIA NOVO, ESPERAR. EU ACHO QUANDO SÁUDE MELHOR, EU CHAMAR MEU AMIG@ SURD@ VOU VISITAR BOM COMUNICAÇÃ̃, MAS FAMÍLIA BARREIRA DE COMUNICAÇÃO NÃO CONSEGUE, DIFÍCIL, MAS É IGUAL CONCORDA? $[\ldots]$

EU TER DESEJO SER LIVRE.

Fonte: elaborado pelas autoras (2021).

$\mathrm{Na}$ entrevista com Pessoa $D$, é possível perceber que ela corrobora os relatos das outras participantes no que concerne à dificuldade de interação, ainda que sejam diferentes em termos de idade, formação, aquisição da língua de sinais, famílias ouvintes com e sem conhecimento da língua de sinais. No trecho "AQUI CASA SENTIR RUIM, LEGAL NÃO", o sentimento de isolamento em ambiente doméstico se apresenta novamente, mas diferentemente do que apontou a Pessoa B, a participante o representa como ruim e do qual não gosta.

A Pessoa $D$ também afirma que o distanciamento social afetou a comunidade surda ao dizer que "ENTÃO, FAMÍLIA MINHA, DESCULPA, OUTRAS FAMÍLIAS NÃO TER LIBRAS, SOZINH@, AINDA NÃO COMUNICAÇÃO, SENTIR SOZINHO, DESEJAR PENSAR AMIGO SURDO CONVIDAR IR JUNTO NÓS DUAS IR TOMAR SORVETE, CONVERSAR, VOLTAR JÁ CONVERSAR UM POUQUINHO, MAS PROBLEMA NÃO TER, EU VEJO PESSOAS ANDANDO NA RUA, SINTO CALMA E SÓ, ENTENDEU?". A necessidade de comunicação parece ser um dos fatores que tornou o distanciamento social ainda mais complexo à comunidade surda, posto que as interações, já limitadas em contextos normais, foram prejudicadas no contexto pandêmico.

No trecho "EU FICO OBSERVANDO O FACEBOOK, VÍDEOS, SURDO FAMÍLIA GRUPO SABE LIBRAS PAI, MÃE SURDO, CRIANÇAS SURDAS TAMBÉM COMUNICAÇÃO, EU VEJO EU SINTO 
EMOÇÃO, OBSERVAR O APRENDIZADO, ENTENDEU? EU GOSTO, ACHO MUITO LEGAL”, a Pessoa $D$ discorre sobre as condições ideais para a integração da pessoa surda à família. Essas condições são pautadas na necessidade de comunicação em uma mesma língua, indicando a demanda de as pessoas ouvintes aprenderem Libras para garantir situações de interação e de aprendizagem no ambiente doméstico.

A solidão no distanciamento social ainda aparece no trecho "DIFÍCIL. ÀS VEZES EM CASA SURDO SOZINHO SENTIR OUTROS PORQUE PARECE PESSOAS MENOSPREZAR, NÃO QUERER PERTO ENTÃO SOZINHO. PRECISA ESPERANÇA, UNIÃO NOSSA FAMÍLIA. DIFERENTE PESSOAS OUVINTES, SAI, VOLTA, PASSEIA, CASA DENTRO PODE PEGAR DOENÇA, PERIGOSO. O SURDO PRECISA RESPEITO SAIR NÃO, PRECISA RESPEITAR, ESPERAR”, em que a Pessoa D compara o comportamento de pessoas ouvintes e pessoas surdas.

Tal sensação é acentuada pela falta de acesso a uma língua de comunicação entre pessoas ouvintes e pessoas surdas dentro da casa, fazendo com que a pessoa surda se sinta menosprezada e solitária, excluída das conversas familiares, como se pode observar no excerto “ENTÃO, EXEMPLO, FESTA AMIG@ (pausa com expressão de tristeza) DIFÍCIL, MAS JÁ ACONTECEU FAMÍLIA MINHA EU SOZINHA, FAMÍLIA INTERAGINDO CONVERSANDO ORALMENTE E EU CHAMAR MINHA IRMÃ PERGUNTAR “O QUE É? ”, MAS ELA "VAI SÓ DEFICIENTE LIBRAS SÓ”, VOLTAR CONVERSAR ORALMENTE. EU AGUENTAR (expressão de tristeza), NÃO SINTO ALEGRIA". A Pessoa D tenta participar da conversação em família, mas o acesso é limitado pela língua, ficando submetida à explicação da irmã, que resume o tópico da conversa, mas não a insere no diálogo. Enfim, a participante diz que aguenta, representando a exclusão linguística como um peso que precisa ser suportado, embora lhe cause tristeza.

Em suma, o que se observa é que a impossibilidade de circular em outros contextos de comunicação projeta sobre o ambiente doméstico uma representação negativa, sustentada pela impossibilidade de interação em língua materna, no caso a Libras, uma vez que as interações são artefatos culturais (STROBEL, 2009).

Essas representações refletem os impactos da naturalização da condição surda como uma "falta", operada pela comunidade ouvinte, que coloca sobre as pessoas surdas a responsabilidade de adaptação à cultura ouvinte e livra as pessoas ouvintes da responsabilidade comunicativa (MOSCOVICI, 2007). Essa condição para a comunicação é representada negativamente pela comunidade surda, fazendo-a refletir sobre a iminente necessidade de a comunicação abarcar sua língua materna tanto nos contextos sociais de trabalho, entretenimento, atendimento a necessidades básicas quanto na relação com a família. 


\section{Considerações finais}

O atual contexto da pandemia da Covid-19 evidenciou um abalo mundial na forma de interação e sociabilidade presencial que constitui um artefato cultural da pessoa surda. Neste estudo, pudemos perceber o quanto as pessoas surdas e surdocegas foram afetadas pelo distanciamento social, bem como observar que as representações sociais negativas direcionadas a esse grupo dificultam sua integração ativa ao mundo social, fazendo-a avançar lentamente. Isso reflete em sua qualidade de vida, seja no contexto familiar ou no extrafamiliar, e tem se agravado no contexto de pandemia do coronavírus.

As análises corroboram o entendimento e a percepção de que o cerceamento de convívio social afeta as pessoas surdas em seu modo de vida, seja em contexto pandêmico ou não. Esse fato é preocupante, por isso é urgente modificar as representações sociais negativas do senso comum e do modelo clínico sobre as pessoas surdas. Entendemos que essa visão resulta na ideia de incapacidade das pessoas surdas, não valorizando suas potencialidades e ressaltando sua falta de audição. Então, sugerimos que a expansão do aprendizado de Libras pode servir como ferramenta de transformação dessa realidade.

Para a Pessoa A e a Pessoa D, o distanciamento social tem duas facetas: o isolamento de ficar em casa e o isolamento de não ter com quem se comunicar dentro da casa por motivos diversos. Nesses dois casos, não há membros da família que dominam a língua de sinais, mas também há casos em que a pessoa surda consegue interagir com a família, é o que experienciam a Pessoa $B$ e a Pessoa $C$. Ainda que a barreira do âmbito familiar seja superada, ela se impõe noutros cenários, como lemos nos relatos da Pessoa $B$ e da Pessoa $C$. O isolamento social foi perceptível nas quatro entrevistas, mas o isolamento linguístico foi identificado apenas em duas, nas entrevistas das Pessoas $A$ e $D$, esta última altamente afetada pelas limitações comunicativas.

Foi possível observar que o isolamento social está diretamente atrelado ao acesso linguístico e que a condição de isolamento pode ser amenizada por meio do atendimento às especificidades comunicacionais da comunidade surda, como o uso de EPI apropriado para comunicação com pessoas surdas oralizadas, por exemplo, e da aprendizagem de Libras para os demais casos. Transpondo-se a barreira linguística, transpõe-se o isolamento.

Acredita-se que uma maneira de mudar essa realidade seja representar as potencialidades de pessoas surdas e surdocegas, ao invés de acentuar a desigualdade social entre elas e as pessoas ouvintes. Também é importante lançar mão de informações sobre manifestações e expressões culturais que destaquem positivamente a comunidade surda e valorizem seus aspectos (uso da língua de sinais, da literatura em Libras, do jeito surdo de compreender o mundo e interagir com ele). De acordo com Moscovici (2007), as representações 
sociais que saem do âmbito particular para o âmbito do senso comum criam uma troca que pode transformar as ideias gerais das pessoas e melhorar a qualidade de vida das pessoas surdas e surdocegas, numa relação de paridade com as pessoas ouvintes.

Em suma, o distanciamento ocasionado pela pandemia de Covid-19 é representado como um evento negativo para a comunidade surda, tanto porque limita seu acesso à comunicação em Libras, quando a família não é usuária da língua, quanto porque afeta o trânsito das pessoas surdas e surdocegas em contextos outros, que lhes permitem interagir e se reconhecerem como comunidade, pela identidade linguística, junto a seus pares.

\section{Referências}

BRASIL. Decreto $n^{0}$ 5.626, de 22 de dezembro de 2005. Regulamenta a Lei $\mathrm{n}^{\circ} 10.436$ de 24 de abril de 2002, que dispõe sobre a Língua Brasileira de Sinais - Libras, e o art. 18 da lei n $^{\circ}$ 10.098 de 19 de dezembro de 2000. Brasília: Congresso Nacional, 2005.

BRASIL. Lei $\mathbf{n}^{\mathbf{0}} \mathbf{1 0 . 4 3 6}$ de 24 de abril de 2002. Dispõe sobre a Língua Brasileira de Sinais — Libras, e o art. 18 da lei $\mathrm{n}^{\circ} 10.098$ de 19 de dezembro de 2000. Brasília: Congresso Nacional, 2002.

BRASIL. Lei $\mathbf{n}^{\mathbf{0}}$ 13.979, de 6 de fevereiro de 2020. Dispõe sobre as medidas para enfrentamento da emergência de saúde pública de importância internacional decorrente do coronavírus responsável pelo surto de 2019. Diário Oficial da União, Brasília, DF, ed. 27, seção 1, p. 1, 7 Fev 2020 [citado em 18 Set 2020]. Disponível em: http://www.in.gov.br/en/web/dou/-/lei-n-13.979-de-6-de-fevereiro-de-2020-242078735. Acesso em: 27 out. 2021.

BUNGEROT, J. et al. The atis sign language corpus. In: Sixth International Conference on Language Resources and Evaluation, 2008. Proceedings. [...] Marrakech, Morocco: European Language Resources Association, 2008, p. 2.943-2946. Disponível em https://aclanthology.org/L08-1470/. Acesso em: 14 dez. 2021.

CAVALIERE, I. A. L.; COSTA, S. G. Isolamento social, sociabilidades e redes sociais de cuidados. Physis: Revista de Saúde Coletiva, Rio de Janeiro, v. 21, n. 2, p. 491-516, 2011.

CARVALHO, M. M. A relação da Libras com a Linguística Sistêmico Funcional (LSF): análise de imagens de sinais com base em variáveis do Contexto de situação. Belas Infiéis, Brasília, v. 9, n. 5, p. 55-73, 2020. DOI: https://doi.org/10.26512/belasinfieis.v9.n5.2020.29368. Acesso em: 14 dez. 2021.

COSTA, L. S. A vida da pessoa com deficiência: reflexões legadas do distanciamento social. In: MENDES, A. et al. (Orgs.). Diálogos sobre acessibilidade, inclusão e distanciamento social: territórios existenciais na pandemia. Rio de Janeiro: Ministério da Saúde, 2020, p. 1011. Disponível em http://www.ideiasus.fiocruz.br/portal/publicacoes-ideiasus/livros/247dialogos-sobre-acessibilidade-inclusao-e-distanciamento-social-territorios-existenciais-napandemia. Acesso em 23 dez. 2020. 
DENZIN, N. K.; LINCOLN, Y. S. A disciplina e a prática da pesquisa qualitativa. In:

DENZIN, N. K.; LINCOLN, Y. S. (Orgs.). O planejamento da pesquisa qualitativa: teorias e abordagens. Porto Alegre: Artmed, Bookman, 2006. p. 15-41.

DICIONÁRIO ON-LINE. Disponível em: https://www.dicio.com.br/. Acesso em 28 out. 2021.

LIMA, R. C. Distanciamento e isolamento sociais pela Covid-19 no Brasil: impactos na saúde mental. Physis: Revista de Saúde Coletiva, Rio de Janeiro, v. 30, n. 2, 2020.

MEDEIROS, A. P.; RAJS, S. As cidades e a pandemia: efeitos, desafios e transformações. In: MENDES, A. et al. (Orgs.). Diálogos sobre acessibilidade, inclusão e distanciamento social: territórios existenciais na pandemia. Rio de Janeiro: Ministério da Saúde, 2020. p. 6-9. Disponível em: http://www.ideiasus.fiocruz.br/portal/publicacoes-ideiasus/livros/247dialogos-sobre-acessibilidade-inclusao-e-distanciamento-social-territorios-existenciais-napandemia. Acesso em: 23 dez. 2020.

MOSCOVICI, S. Representações sociais: investigações em psicologia social. 5. ed. Trad. Pedrinho A. Guareschi. Petrópolis: Vozes, 2007.

PATROCÍNIO, P. R. T. Notas sobre narrativas autobiográficas de autores surdos. Revista Araticum, v. 21, n.1, P. 91-103, 2020. Disponível em: https://doi.org/10.46551/2179679320200006. Acesso em: 14 dez. 2021.

PERLIN, G.; MIRANDA, W. Surdos: o narrar e a política. Ponto de vista, n. 5, p. 217-226, 2003.

QUADROS, R. M. Libras. São Paulo: Parábola, 2019.

QUADROS, R. M. O tradutor e intérprete de língua brasileira de sinais e língua portuguesa. Programa Nacional de Apoio à Educação de Surdos, Brasília: MEC; SEESP, 2004.

REIS, R.; GATI, H.; MACHADO, M. M. Isolamento social dos surdos em organizações. In: XXII SemeAd - Seminários em Administração, 2019. Anais. [...]. São Paulo: FEA/USP, p. 1$16,2009$.

SANTOS JUNIOR, E. O indivíduo surdo na sociedade ouvinte: resistir é preciso. Revista Virtual de Cultura Surda, n. 27, p. 1-16, março de 2020.

STROBEL, K. As imagens do outro sobre a cultura surda. 2. ed. rev. Florianópolis: Editora da UFSC, 2009.

TELESSAÚDERS. Página virtual. Disponível em: https://www.ufrgs.br/telessauders/posts_coronavirus/qual-a-diferenca-de-distanciamentosocial-isolamento-e-quarentena/. Acesso em: 27 out. 2021.

VIEIRA, V.; RESENDE, V. M. Análise de discurso (para a) crítica: o texto como material de pesquisa. 2. ed. Campinas, São Paulo: Pontes Editores, 2016. 
WILCOX, S.; WILCOX, P. P. Aprender a ver. Rio de Janeiro: Editora Arara Azul, 2005.

\section{Sobre as autoras}

Marília do Socorro Oliveira Araújo (Orcid iD: https://orcid.org/0000-0002-2386-3832)

Mestranda do Programa de Pós-Graduação em Cidades, Territórios e Identidades da Universidade Federal do Pará (UFPA); licenciada em Letras - Língua Portuguesa pela mesma instituição; especialista em Libras: Docência e Interpretação e em Língua Portuguesa e Literatura pela Faculdade de Educação e Tecnologia da Amazônia (FAM). É intérprete pela Associação de Tradutores/Intérpretes de Língua de Sinais do Pará (ASTILP). Bolsista FAPESPA.

Márcia Monteiro Carvalho (Orcid iD: https://orcid.org/0000-0001-6278-2667)

Doutora em Estudos da Tradução pela Universidade Federal de Santa Catarina (UFSC); mestra em Letras: Estudos Linguísticos pela Universidade Federal do Pará (UFPA); especialista em Docência de Libras (FTED). É intérprete pela Associação de Tradutores/Intérpretes de Língua de Sinais do Pará (ASTILP). Líder do grupo de pesquisa Discurso e Relações de Poder (DIRE/UFPA/CNPq). É professora da Universidade Federal do Pará e do Programa de Pós-Graduação em Estudos da Tradução da UFSC.

Rosângela do Socorro Nogueira de Sousa (https://orcid.org/0000-0003-0429-9700) Doutora em Linguística pela Universidade Federal do Ceará (UFC); mestra em Letras: Estudos Linguísticos pela Universidade Federal do Pará (UFPA); especialista em Língua Portuguesa: uma abordagem textual pela mesma instituição. Líder do grupo de pesquisa Discurso e Relações de Poder (DIRE/UFPA/CNPq). Professora do Programa de PósGraduação em Cidades, Territórios e Identidades da UFPA.

Recebido em junho de 2021.

Aprovado em dezembro de 2021. 